\title{
¿Cómo desarrollar secuencialmente el conocimiento fonológico?
}

\section{Ana Belén Dominguez y María Clemente}

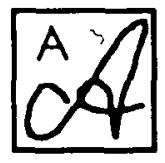

$\mathcal{D}$

A partir de una revisión de los procesos que requiere la "conciencia fonológica", necesaria para la lectura, se proponen aqui una serie de actividades para desarrollarla.

\section{INTRODUCCION}

El aprendizaje de la lectura y de la escritura es una de las mayores preocupaciones y uno de los retos más importantes con los que se enfrentan los profesionales de la enseñanza de los primeros niveles educativos, por lo cual, cuestiones referidas al hecho de cómo aprenden los niños a leer y escribir y, especialmente, cómo enseñar a leer y escribir, son algunas de las principales inquietudes de estos profesionales; a la vez que tienen un gran interés desde el punto de vista de la investigación en lectura. Un hecho que nos ayudaría a resolver, en parte, estas cuestiones sería descubrir cuáles son las habilidades de las que dependen los niños para aprender a leer y escribir, ya que, si sabemos qué habilidades son necesarias podremos ayudar a los niños a desarrollarlas.

En este sentido, el enfoque tradicional sobre el aprendizaje de la lectura, que ha influido y se ha reflejado en la práctica escolar de los docentes durante mucho tiempo, considera las habilidades perceptivomotoras, tales como la lateralidad, el esquema corporal, la discriminación visual, la coordinación visomotora, etc., como prerrequisitos para 


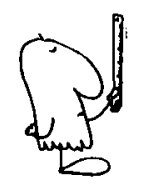

aprender a leer y escribir (y como causas de las dificultades en dichos aprendizajes). Sin embargo, durante las últimas décadas muchos han sido los avances que se han hecho dentro del campo de la lectura. Numerosos trabajos, fundamentalmente desde una perspectiva psicolingüística, han cuestionado y criticado los planteamientos perceptivomotores y han resaltado la importancia de las habilidades de análisis fonológico como una de las capacidades críticas en el aprendizaje de la lectura y de la escritura. Estos trabajos (p.e., Ellis, 1981; Vellutino, 1979) demuestran claramente que los niños con dificultades en el aprendizaje de la lectura no tienen problemas de percepción visual ni viso-espacial, sino que sitúan el problema en deficiencias en el propio lenguaje del niño. Así, las dificultades pueden surgir no tanto a la hora de analizar las formas visuales de los grafemas, como al convertir -traducir-esos grafismos en unidades fonológicas o en lenguaje. Según este punto de vista, la dificultad para aprender a leer no reside en distinguir los símbolos gráficos que contiene el sistema alfabético, sino en comprender lo que se representa con cada uno de ellos, es decir, los problemas no estarían en distinguir visualmente la letra $d$ de la letra $b$, sino en constatar la presencia del fonema /b/y el /d/ en el propio lenguaje.

Como consecuencia de este planteamiento se deduce la importancia que realmente tiene el conocimiento fonológico en el proceso de aprendizaje de la lectura y de la escritura, y la necesidad de sensibilizar al niño hacia ese conocimiento, o en términos más concretos, ayudarle a romper la palabra en sílabas y fonemas, identificar sonidos comunes, contar los sonidos, tomar conciencia de cómo y dónde se articulan, etc. Todo ello debido al hecho de que cuando un lector experimentado lee o escribe (o cuando un niño aprende a leer o escribir) puede hacer uso de la información fonológica que tiene sobre su propio lenguaje, hecho que se ha denominado como procesamiento fonológico.

Numerosas investigaciones (ver Wagner y Torgesen, 1987 para una revisión) han centrado su interés en el análisis del procesamiento fonológico que opera cuando un lector se enfrenta a lo escrito en un sistema alfabético y muy especialmente en el conocimiento fonológico o capacidad de operar explícitamente con los segmentos menores del habla, con el fin de comprobar la relación que tiene ese conocimiento con el aprendizaje de la lectura. A partir de estos estudios, se ha comprobado que este conocimiento fonológico está fuertemente relacionado con el éxito alcanzado en lectura (ver p.e., Morais, Alegria y Content, 1987; Goswami y Bryant, 1990; Wagner y Torgesen, 1987), pero aún no se ha comprendido totalmente cuál es el papel preciso que juegan estas habilidades fonológicas y cuáles serían los métodos implicados en su enseñanza. Diversos estudios (p.e., Bradley y Bryant 1983; Byrne y Fielding-Barnsley, 1991; Cunningham, 1990; Lundberg, Frost y Petersen, 1988) han demostrado que el entrenamiento de los niños en habilidades de análisis fonológico produce mejoras en el aprendizaje de la lectura. Estos estudios ponen de manifiesto que las habilidades fonológicas son un prerrequisito útil, e incluso necesario, para el éxito en el aprendizaje de la lectura. Por ello, tiene sentido incluir la enseñanza de 
habilidades fonológicas en las etapas tempranas del currículum de lectura; esto es, deberíamos intervenir de tal forma que los niños percibieran la estructura fonológica del lenguaje oral, para lo cual, resulta previo que sean capaces de distinguir las unidades en las que se puede descomponer el lenguaje hablado: oraciones, palabras, sílabas y, muy especialmente, fonemas. Es decir, es preciso desarrollar en los niños la habilidad de centrar la atención sobre el lenguaje y reflexionar sobre su naturaleza y estructura, capacidad que tiene un importante papel en el aprendizaje de la lectura y de la escritura y que se conoce como conciencia metalingüistica.

Si esto es así, desde un punto de vista educativo, la cuestión que nos interesaría resolver sería: ¿Cómo podríamos ayudar a los niños a desarrollar este conocimiento fonológico?. Las investigaciones realiza. das sobre el conocimiento fonológico nos proporcionan alguna guía para la selección de tareas y/o actividades de enseñanza-aprendizaje que podrían tener cabida en un currículum de Educación Infantil. En este artículo discutimos algunas de las implicaciones educativas de estas investigaciones, a la vez que proponemos actividades que incorporan los principios teóricos analizados por dichos trabajos, señalando, a su vez, algunos aspectos que deben ser tenidos en cuenta en esta selección de tareas $y / 0$ actividades, y que fundamentalmente proceden de un trabajo que hemos llevado a cabo (Domínguez, 1992), aunque también aludiremos a otras investigaciones efectuadas en esta línea.

\section{ACTIVIDADES PARA EL DESARROLLO FONOLOGICO: CRITERIOS PARA SU DISEÑo}

Revisando las investigaciones efectuadas hasta el momento observamos un amplio y variado número de tareas que han sido empleadas para evaluar, medir o entrenar en conocimiento fonológico, p.e.: reconocer una rima; identificar, contar, invertir, combinar, añadir, sustituir y omitir fonemas (ver Content, 1985; Lewkowicz, 1980; Yopp, 1988 para una revisión); tareas que, básicamente y con las pertinentes modificaciones, pueden servirnos para la enseñanza dentro del aula. Los datos procedentes de estas investigaciones nos muestran también que la dificultad de las tareas que miden el conocimiento fonológico depende del nivel lingüistico de la unidad de palabra que se quiera trabajar, esto es, rima, sílaba, y fonemas (Treiman, 1992), de tal manera que a mayor nivel de abstracción mayor dificultad tendrá la tarea. De los requisitos cognitivos que los niños tienen que poner en juego para realizar con éxito tal tarea (Lundberg, Frost y Petersen, 1988; Tunmer y Rohl, 1991), siendo mayor la complejidad cuantos más sean los mecanismos cognitivos que se pongan en juego. Y por último, de la necesidad de una enseñanza explicita.

El nivel lingüístico más sencillo y el que precisa una menor carga cognitiva sería la rima, requiriendo una manipulación de segmentos menos consciente y deliberada que los otros niveles (Lundberg, 1978; 
Stanovich y col., 1984), además, el conocimiento de la rima no parece depender mucho de una enseñanza formal. El nivel silábico sería el segundo en orden de complejidad, ya que, para darse cuenta de su existencia los niños pueden apoyarse en unidades articulatorias naturales y, por ello, el conocimiento silábico sería más asequible para los niños de Educación Infantil que el de los fonemas, dependiendo menos de una enseñanza sistemática para su desarrollo, siendo suficiente en la mayoría de los casos un mínimo de ayuda para que los niños manipulen sílabas (ver Mann, 1986). El nivel de mayor complejidad lingüística y cognitiva sería el fonológico, siendo precisa una instrucción específica para que los niños adquieran el conocimiento fonológico, ya que no surge espontáneamente (ver Morais, Alegría y Content, 1987). La consecuencia educativa que se deriva de esta afirmación es que llegado el momento debemos ayudar a los niños a través de una enseñanza explícita a descubrir la estructura segmental de la palabra, propia de los sistemas de escritura alfabéticos. Para lo cual propondremos una serie de actividades que ayuden a descubrir esta estructura, antes de lo cual vamos a considerar algunos aspectos basados fundamentalmente en nuestra investigación (Domínguez, 1992) y que hacen referencia a los siguientes aspectos: 1) cuál es la secuencia de tareas más apropiada para llegar a captar la estructura fonológica del lenguaje; 2) la relevancia o no de trabajar las unidades de la palabra en posiciones iniciales, medias o finales; 3) en qué orden deben trabajarse los grupos fonéticos; 4) la conveniencia de trabajar con distintos tipos de sílabas. Vamos a comentar más detenidamente cada uno de ellos.

\section{SECUENCIA DE TAREAS}

Es posible establecer una secuencia de aprendizaje de las tareas que conducen à los niños a analizar las palabras, en función de su complejidad. Así, entre las diversas tareas consideradas en nuestra investigación la secuencia, de más fácil a más compleja, sería: rima, identificación, adición y omisión.

Por tanto, la primera tarea que podríamos emplear para ayudar a los niños de Educación Infantil a desarrollar un conocimiento de la estructura sublexical de la palabra sería la rima. En esta tarea pedimos a los niños que determinen si dos o más palabras comparten al final un mismo grupo de sonidos (p.e., botella/estrella; pato/gato/plato). Existen investigadores que señalan que el conocimiento fonológico puede desarrollarse inicialmente a través de un conocimiento de la rima, el cual puede, incluso, facilitar el posterior aprendizaje de la lectura (Bradley y Bryant, 1991; Goswami y Bryant, 1990). Sin embargo, esta tarea no implica la segmentación explícita de las palabras en fonemas separados (Morais, 1991; Morais, Alegria y Content, 1987). Dentro de nuestro trabajo, la tarea de rima se mostró poco rentable a la hora de incrementar los niveles de conocimiento fonológico de los niños, aunque pensamos que esta tarea puede ser útil en los momentos iniciales, por su atractivo y su facilidad, para que los niños presten atención a los sonidos del lenguaje, o más bien, para desarrollar en ellos la habilidad para descen- 
trarse del significado y atender a la forma fonológica del lenguaje (p.e., palabras que significan cosas diferentes tienen sonidos iguales al final); lo que puede llevar a los niños a centrarse en la similitud (es decir, la rima) y en la diferencia (es decir, el comienzo) entre las palabras.

Siguiendo la secuencia de aprendizaje establecida anteriormente, después de la rima, utilizaríamos la tarea de identificación. En esta tarea se le pide a los niños que juzguen si un sonido determinado se halla en una o varias palabras en alguna posición, esto es, comienzo, medio o final de la palabra (p.e., libro/flor/sol). En la revisión de las investigaciones, encontramos autores (Byrne y Fielding-Barnsley, 1990, 1991) que han utilizado esta tarea para el desarrollo del conocimiento fonológico de los niños. En nuestra investigación la actividad de identificación (en concreto de fonemas) fue relativamente fácil de enseñar a los niños y, a través de ella, logramos incrementar sus niveles de conocimiento fonológico. Además, esta tarea puede ser útil para enseñar a los niños el sistema de correspondencias grafema-fonema, ya que, una vez que los niños se percaten de la existencia de los sonidos en su lenguaje oral podemos mostrarles la letra que los representa en el lenguaje escrito. Por ello, pensamos que, con este conocimiento de las correspondencias letra-sonido, la tarea de identificación de fonemas puede ser un buen vehículo para favorecer en los primeros momentos de la enseñanza el desarrollo de habilidades fonológicas en los niños prelectores.

La tarea que podríamos plantear a continuación dentro de un programa de enseñanza sería la adición. En ella, la tarea del sujeto consiste en añadir un segmento determinado (sílaba o fonema) a una palabra o pseudopalabra (serie de letras pronunciables pero sin significado). El educador dice una palabra o pseudopalabra al niño y le pregunta qué nueva palabra se forma una vez añadido el segmento indicado por el mismo (p.e., $/ \mathrm{p} /+$ lato; moto $+/ \mathrm{r} /$ ). Los resultados obtenidos en nuestra investigación en cuanto su eficacia para el desarrollo del conocimiento fonológico fueron equivalentes a los de la tarea de omisión, por lo cual los comentaremos conjuntamente, si bien hay que resaltar que la adición se mostró algo más fácil de realizar. También encontramos dentro de las investigaciones realizadas autores que han empleado este tipo de tarea (Morais y col., 1979; Read y col., 1986; Rueda, Sánchez y González, 1990).

Dentro de la secuencia de aprendizaje que hemos establecido, la última tarea que emplearíamos sería la omisión, en la cual los niños deben omitir un segmento (sílaba o fonema) de una palabra o pseudopalabra y decir que es lo que queda (p.e., pala - /p/; arañar - /r/). Esta tarea ha sido usada con bastante frecuencia para evaluar el conocimiento fonológico de los niños (Bruce, 1964; Calfee, Lindamood y Lindamood, 1973; Morais, Cary, Alegria y Bertelson, 1979) o para desarrollarlo a través de un entrenamiento explícito (Cunningham, 1990). En nuestro trabajo, la omisión de fonemas fue la más compleja de realizar, pero la que mayores niveles de habilidad fonológica creó en los niños, junto a la adición; niveles que se incrementan (tal y como señalan Byrne y Fielding-Barnsley, 1991; Stuart y Coltheart, 1988) si combinamos esta 
enseñanza con el conocimiento de las letras del alfabeto. Además, fue la tarea que situó a los niños en mejores condiciones de cara al posterior aprendizaje de la lectura y de la escritura. Otro hecho importante, que también observamos a través de nuestra investigación, fue que esta tarea es muy útil para ayudar a los niños a analizar sílabas del tipo CCV (p.e., pla, tra, etc.) en los fonemas que las componen y con ello reducir los problemas que suelen presentarse a la hora de leer y escribir estas sílabas. Por lo tanto, pensamos que esta tarea, a pesar de la dificultad inicial que presenta, debe incluirse siempre en el contexto de la preparación de los niños de Educación Infantil para el aprendizaje de la lectura y de la escritura.

\section{POSICION DE LA UNIDAD DE ANALISIS DENTRO DE LA PALA- BRA}

El segundo aspecto a tener en cuenta en la selección de actividades destinadas a desarrollar en los niños de Educación Infantil habilidades de análisis fonológico, sería la posición de la palabra en la cual vamos a pedir a los niños que identifiquen, añadan y omitan un segmento (sílaba o fonema), esto es, al comienzo, en el medio o al final de la palabra. Los resultados de nuestra investigación muestran que la enseñanza destinada a desarrollar habilidades de análisis de la palabra es igual de efectiva si utilizamos sonidos iniciales, medios o finales. La postura moderada sería usar todas la posiciones, aunque nuestros datos (al igual que los de otros autores, p.e., Byrne y Fielding-Barnsley, 1990) señalan que la enseñanza se generaliza a otras posiciones de la palabra de aquella en la cual fue aprendida. En cualquier caso, a partir del análisis que efectuamos tarea por tarea, observamos que es más fácil para los niños de estas edades identificar sílabas y fonemas situados al comienzo de la palabra que al final, y ambas actividades son más fáciles que identificar fonemas en medio de palabra; mientras que, las tareas de adición y de omisión (de sílabas y de fonemas) son más sencillas para los niños cuando les pedimos que las realicen en posición final de palabra que al comienzo, e igualmente, ambas posiciones son más fáciles que la de en medio de la palabra.

\section{GRUPOS FONÉTICOS}

Otro aspecto a considerar en esta selección sería el tipo de fonemas que vamos a utilizar para llevar a cabo esta enseñanza fonológica. Los resultados de nuestro trabajo muestran, por un lado y por lo que respecta a los fonemas consonánticos, que las fricativas son más útiles en los momentos iniciales de enseñanza que las consonantes oclusivas (aunque generalmente en el orden de las adquisiciones fonológicas las fricativas son posteriores a las oclusivas una vez que el nivel lingüístico del pequeño está adquirido, las fricativas son más fáciles de aislar en la corriente acústica que las oclusivas, razón por la cual proponemos que se trabaje en primer lugar con las consonantes fricativas). $Y$, por otro lado, que no parece necesario tener que trabajar todos y cada uno de los fonemas, ya que, la haviìdad de análisis fonológico también se 
generaliza hacia otros fonemas que no han intervenido en la enseñanza; por ello, podría ser suficiente una subserie de fonemas que representen las diversas clases.

\section{ESTRUCTURA DE LAS SILABAS}

Finalmente, otro aspecto a tener en cuenta sería el tipo de sílabas presentes en las palabras que vamos a emplear para enseñar a los niños a realizar un análisis fonológico de las mismas. Dentro de esta enseñanza debemos contemplar todo tipo de sílabas, prestando especial atención a aquellas que contienen grupos consonánticos (CCV), ya que, en nuestra investigación conseguimos, a través de la enseñanza fonológica explícita, que los niños fueran capaces de analizar este tipo de sílabas en los fonemas que las componen, y con ello se redujeron las dificultades a la hora de leer y escribir estas sílabas (resultado que concuerda con el obtenido por Treiman, 1991).

\section{PROPUESTA DE ACTIVIDADES}

La pretensión que tenemos al proponer actividades para desarrollar en los niños de la escuela infantil el conocimiento fonológico es la de dar ejemplos, más que la de presentar un programa exhaustivo que sobrepasaría los límites de un artículo. Las bases en las que las sustentamos han sido descritas más arriba y tan sólo queremos establecer ahora los criterios que nos parecen necesarios tener en cuenta para su realización didáctica.

En primer lugar, cualquier actividad debe contemplarse dentro del proyecto global y contextual de cada centro o aula. En segundo lugar, el orden secuencial de las tareas será el que se deriva de las investigaciones que hemos ido analizando, aunque sin duda muchas tareas deberán repetirse y volver sobre ellas. En tercer lugar, los criterios que deben observarse deberán ser interpretados por el profesor buscando actividades que tengan en cuenta quizá más de uno, aunque necesariamente a la hora de precisarlos por nuestra parte debemos presentarlos ordenada y esquemáticamente para su mejor comprensión. Estos criterios para la selección de actividades destinadas a desarrollar en los niños el conocimiento metalingüístico en el nivel fonỏógico son los siguientes:

1) trabajáremos indistintamente tanto el análisis desde la frase hasta el fonema, como jugando directamente con palabras;

2) igualmente podemos iniciar el proceso con tareas más básicas tales como por ejemplo ejercicios de discriminación auditiva (de sonidos en general), pero con la seguridad de que estos ejercicios no son más que un paso previo sin una rẹlación relevante con lo fonológico;

3) el orden que proponemos estará marcado por los siguientes aspectos, que ya fueron expuestos anteriormente y que resumimos a continuación:

- Respecto de las tareas: rima, identificación, adición u omisión. 


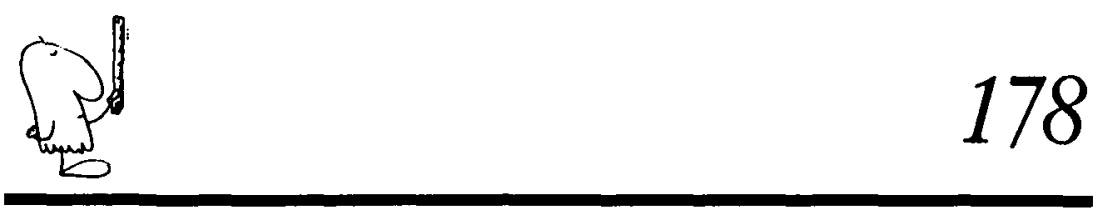

- En relación con la posición de las unidades de análisis dentro de la palabra es indistinto trabajar con sonidos iniciales, medios o finales.

- Por lo que se refiere a los grupos fonéticos parece aconsejable trabajar primero las vocales, luego las fricativas y las oclusivas.

En relación con estos dos últimos aspectos dado que la habilidad metalinguística se generaliza tanto desde unos grupos fonéticos a otros como desde unas posiciones dentro de la palabra a otras, no será necesario trabajar con todos los fonemas ni en todas las posiciones, pudiendo seleccionar una serie de fonemas que sean representativos. Sin embargo, en relación con la estructura de las sílabas (CV, $\mathrm{CVC}, \mathrm{CCV}$, etc.) si parece necesario trabajar con todas y cada una de ellas.

Tomando como referencia estos criterios proponemos una serie de ejemplos de ejercicios clasificados por tareas.

\section{Ejemplos de Actividades de Rima}

- Damos a los niños una serie de dibujos preparados de antemano, para que agrupen aquellos que riman.

- Puestos en corro, decimos una palabra y los niños dirán por orden otra que "termine igual".

\section{Ejemplos de Actividades de Identificación de Silabas}

- Juego del Veo-Veo.

- Juego del Tren de Palabras (cada niño dice una palabra que empiece igual que aquella por la que terminaba la que ha dicho el anterior).

- Se presenta un reloj de cartulina que contenga sílabas, se hace girar la aguja y cuando se detenga los niños deberán decir; a) palabras que empiecen por la sílaba señalada; b) palabras que terminen por ésta. (Este mismo ejercicio puede realizarse sólo con fonemas).

\section{Ejemplos de Actividades de Identificación de Fonemas}

- Identificar en una lámina dibujos cuya palabra empieza o termina por un determinado fonema.

- Trabajar con dominos de dibujos con el fin de que los niños busquen un dibujo cuyo primer fonema sea el mismo que aquel por la cual termina la ficha anteriormente puesta.

(La identificación de fonemas puede trabajarse con ejemplos similares a los de la identificación de sílabas, pero ya que aislar el fonema no es una intuición natural en los niños como es aislar la sílaba deberemos propiciar ayudas para que consigan realizarlo, p.e.: al decir la palabra exagerar mucho el sonido inicial, pidiendo a los niños que digan cuál es el sonido que han oído durante más tiempo y lo comparen con el sonido que se este trabajando, preguntando, p.e.,: "isuenan igual el primer sonido de /ssssol/ y /ssss/?"). 
Ejemplos de Actividades de Adición u Omisión de Stlaba

- Colocamos a los niños en dos grupos, uno en frente del otro, les decimos a un grupo sílabas y al otro palabras o pseudopalabras, y les pedimos que se junten por parejas de manera que formen una palabra nueva o pseudopalabra.

- Presentamos dos columnas de sílabas. En cada fila de la primera sólo hay una sílaba y en cada fila de la segunda puede haber varias. Los niños deben unir con una flecha la sílaba de la primera columna con las de la segunda, a fin de formar palabras. Por ejemplo:

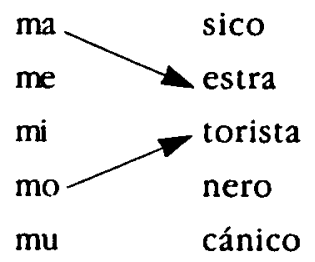

- Juego de supresión de sílabas: se da una lista de palabras de tres sílabas y los niños deberán formar nuevas palabras suprimiendo alguna sílaba. Por ejemplo: Fábula — bula; pescado — pesca; solfeo - sol, feo.

\section{Ejemplos de Actividades de Adición u Omisión de Fonemas}

- Se presenta a los niños una marioneta que "habla de forma curiosa, con pedazos pequeños", y que le gusta inventar palabras que a veces no quieren decir nada (sin significado). Se demuestra cómo habla la marioneta: produciendo el fonema inicial aislado del resto de la palabra (p.e., "sol" - " $/ s / .$. ol"), pidiendo a los niños que lo imiten. Se Juega con la marioneta a inventar palabras que no significan nada, diciendo, p.e.: "si a 'sol' le quitamos o no decimos /s/ me queda ..." (los niños lo imitan). Para facilitar la actividad se puede dar la siguiente ayuda: decir la palabra segmentada alargando o repitiendo el fonema inicial en el momento en que los niños deben omitir el fonema: p.e., "si a 'ssss... ol' le quitamos o no decimos /ssss/ me queda ...". 


\section{Referencias}

Bradley, L. y Bryant, P.E. (1983). "Categorizing sounds and leaming to read a causal connection". Nature, 301, 419-421.

Bradiey, L. y Bryant, P.E. (1991). "Phonological skill before and after learning to read". En S.A. Brady y D.P. Sankweiler (Eds.). Phonological process in literacy. Hilldale: L.E.A.

BRUCE D.J. (1964). "The analysis of word sounds". British Joumal of Educational Psycbology, 34, $158-170$.

BYRNE, B. y FIELDING-BARnSLEY, R. (1990). "Acquiring the alphabetic principle: A case for teaching recognition of phoneme identity". Joumal of Educational Psycbology, 82(4), 805-812.

BYRNE, B. y FiELDING-BARNSLEY, R. (1991). "Evaluation of a program to teach phonemic awareness to young children". Joumal of Educational Psycbology, 83 (4), 805-812.

Calfee, R.; Lindamood, P. y Lindamood, C. (1973). "Acoustic-phonetic skills and reading: Kindergarden through twelfth grade". Jourmal of Educational Psycbology, 64, 293-298.

CONTENT, A. (1985). "Le développement de l'habileté d' analyse phonétique de la parole". $L$ 'année Psychologique, 85, 73-99.

Cunningham, A.E. (1990). "Explicit versus implicit Instruction in Phonemic Awareness" Journal of Experimental Child Psycbology, 50, 429-444.

DOMINGUEZ, A.B. (1992). La enseñanza de babilidades de analisis fonológico en el aprendizaje de la lectura y de la escritura. Programas para Educaión Infantil. Tesis Doctoral. Universidad de Salamanca.

EuIs, A. (1981). "Visual and name coding in dyslexic children". Psychological Research, 43, 201218.

GosWant, U. y Bryant, P. (1990). Phonological Skills and Leaming to Read. London: Lawrence Erlbaum.

LEwKowncz, N.K. (1980). "Phonemic Awareness Training: What to Teach and How to Teach It" Journal of Educational Psycbology, 72 (5), 686-700.

LUNDBERG, 1. (1978). “Aspects of linguistic awareness related to reading. En A. Sinclair; R.J. Jarvella y W.J. Levelt (Eds.). The child's conception of language. Berlín: Springer-Verlag. (p. 83-96).

Lundaerg, I.; Frost, J. y Petersen, O. (1988). "Effects of an extensive program for stimulating phonological awareness in preschool children". Reading Research Quarterly, 23, 256-284.

MaNn, V.A. (1986). "Phonological awareness: The role of reading experience". Congition, 24, 6592.

Morais, J. (1991). "Phonological awareness: A bridge between language and literacy". En D.J. Sawyer y B.J. Fox (Eds.). Pbonological awareness in reading. New York: Springer-Verlag.

MORAIS, J.; AleGRu, J. y CONTENT, A (1987). "The relationships between segmental analysis and alphabetic literacy: an interactive view". Cabiers de Psycbologie Cognitive, 7 (5), 1-23.

MORAS, J.; CARY, L.; ALEGRIA, J. y BERTELSON, P. (1979). "Does awareness of speech as a sequence of phones arise spontaneously?". Cognition, 8, 1-9.

READ, C.; ZHANG, Y.; NIE, H. y DING, B. (1986). "The ability to manipulate speech sounds depends on knowing alphabetic reading". Congition, 24, 31-44.

Rrueda, M.l.; SANCHEz, E. y Gonzalez, L. (1989). "El análisis de la palabra como instrumento para la rehabilitación de la dislexia". Infancia y Aprendizaje, 49, 39-52.

Stanovich, K.E.; Cunningham, A.E. y Cramer, B.B. (1984). “Assessing phonological awareness in kindergarten children: Issues of task comparability" Joumal of Experimental Cbild Psycbology, 38, 175-190.

StunRt, M. y COLThenrt, M. (1988). "Does reading develop in a sequence of stages?". Cognition, 30, 139-181.

TREIMAN, R. (1991). "Children's spelling errors on syllable-initial consonant clusters". Joumal of Educational Psycbology, 83 (3), 346-360.

Treiman, R. (1992). "The role of intrasyllabic units in learning to read". En L.C. Ehri y R. Treiman (Eds.). Reading acquisition. Hilladale: L.E.A. 
TUNMER, W.E. y ROHL, M (1991). "Phonological awareness and reading acquisition". En D.J. Sawyer y B.J. Fox (Eds.). Pbonological awareness in reading. New York: Springer-Verlag.

Velutino, F. (1979). Dyslexia: Theory and research. Cambridge: Mass., M.I.T. Press.

WAGNER, R.K \& TORGESEN, J.K. (1987). "The nature of phonological processing and its causal role in the acquisition of reading skills". Psycbological Bulletin, 101 (2), 192-212.

YoOP, H.K. (1988). "The validity and reliability of phonemic awareness tests". Reading Researcb Quarterly, 23, 2, 159-177.

\section{¿Cómo desarrollar secuencialmente} el conocimiento fonológico?

\section{Ana Belén Dominguez y María Clemente Linuesa CL\&E, 1993, 19-20, 171-181}

Resumen: El conocimiento fonológico o conciencia fonológica se ha idenificado como una habilidad clave para aprender a leer en los sistemas alfabéticos; que el niño adquiera ese conocimiento es sin duda una valiosa ayuda para facilitar el aprendizaje lector. En este artículo se analizan algunos aspectos ampliamente estudiados como son la habilidad para reconocer la rima de las palabras, la conciencia silábica y el reconocimiento de fonemas y su papel en el desarrollo del conocimiento fonológico. Finalmente como consecuencia de este análisis se proponen una serie de ejemplos de actividades para ese desarrollo

Datos sobre las autoras: Ana Belén Domínguez Gutierrez es profesora ayudante de Didáctica y María Clemente Linuesa, profesora titular de Didáctica del Departamente de Didáctica, Organización y Métodos de Investigación de la Facultad de Educación de la Universidad de Salamanca. Ambas autoras han trabajado durante los últimos años en los aspectos psicopedagógicos de la lectura.

Dirección: Departamento de Didáctica, Organización y Métodos de Investigación. Facultad de Educación. Universidad de Salamanca. Paseo : Canalejas, 169; 37008 Salamanca

(1) De todos los artículos deberá solicitarse por escrito autorización de CL\&E y de los autores para el uso en forma de facsímil, fotocopia o cualquier otro medio de reproducción impresa. C1\&E se reserva el derecho de interponer acciones legales necesarias en aquellos casos en que se contravenga la ley de derechos de autor. 